\title{
Lipase-Catalyzed Polymerization of Glycerol and Dicarboxylic Acids in an Organic Medium
}

\section{Romina C. Pessagno and Alicia Baldessari}

Departamento de Química Orgánica, Facultad de Ciencias Exactas y Naturales, Universidad de Buenos Aires, Pabellón 2, $3^{\circ}$,Ciudad Universitaria, 1428 - Buenos Aires, Argentina

E-mail: alib@qo.fcen.uba.ar

\begin{abstract}
Lipases from different sources catalyze the polyesterification of glycerol and several dicarboxylic acids in presence or organic solvents
\end{abstract}

\section{Introduction}

Enzymatic polymerizations are receiving much attention as a new method for polymer production since biocatalysis is expected to generate environmentally acceptable properties such as biodegradability and biocompatibility [1]. The opportunities available in the use of enzymes in polymer science include enhanced control of regioselectivity, enantioselectivity, molecular weight and dispersity and the ability to synthesize entirely new polymers [2].

Lipases in organic solvents are efficient catalysts in polyesterification [3] and oligomerization reactions. In these polymerizations, the use of traditional chemical catalysis is limited because catalysts tend to have an undesirable effect on the subsequent polymerization reactions.

Lipase-catalyzed condensations reported in literature describe use of hydroxyacids and diols as hydroxylated monomers, but at this moment poliols, such as glycerol, have not been investigated [3]. Here we report the lipase-catalyzed regioselective polyesterification of glycerol and several carboxylic acids.<smiles>COCC(O)COC(=O)[In](C)C(=O)I</smiles>

R: - $\left(\mathrm{CH}_{2}\right)_{2^{-}} ;-\left(\mathrm{CH}_{2}\right)_{8^{-}} ;-\mathrm{C}_{6} \mathrm{H}_{4^{-}}(\mathrm{o}$ and $\mathrm{p})$ 


\section{Experimental}

The procedure involved addition of enzyme to a solution of glycerol and the carboxylic acid in the appropriate solvent with molecular sieves $0.4 \mathrm{~nm}$ when indicated. The suspension was held at $200 \mathrm{rpm}$ and $30^{\circ} \mathrm{C}$ for $72 \mathrm{~h}$. Disappearence of monomers was monitored by TLC. The enzyme was filtered off and washed with solvent. The filtrate was evaporated in vacuo and the resultant polymer was dried. The product was analyzed by UV-MALDI-TOF-MS, NMR and FTIR.

\section{Results and Discussion}

Enzymatic polymerization was performed under different experimental conditions. Lipase-catalyzed polymerization was studied with several enzymes such as porcine pancreatic lipase, Candida rugosa lipase, Mucor miehei lipase and Candida antarctica lipase (CAL). Best results were obtained with CAL Different solvents were used: dioxane, tetrahydrofuran, etc.

Average number $\left(M_{n}\right)$ (1695-1979) and weight average $\left(M_{w}\right)$ (1704-2110) molecular masses of products obtained by polymerization in presence of molecular sieves were calculated from UVMALDI-TOF-MS spectra.. Spectra of lipase-catalyzed polymers showed a remarkable monodispersity (Dp: 1.01-1.07) which is difficult to achieve by conventional polymerization procedures. $200 \mathrm{MHz}{ }^{1} \mathrm{H}$ NMR spectra of polymers showed broad signals between 4.10 and $4.30 \mathrm{ppm}$. Lower field signals were not observed indicating that secondary hydroxyl is not esterified and that lipase catalyzes polycondensation in a regioselective way. Different experimental conditions changed molecular weight of polymers but not its regioselectivity. Highest molecular weights were obtained by withdrawing water from the system with molecular sieves and anhydrous dioxane as solvent. Without molecular sieves only oligomers were obtained.

In summary, the lipase-catalyzed polyesterification here presented provides a simple, regioselective and economical method for preparing polyhydroxylated low molecular weight polyesters from glycerol and dicarboxylic acids. This enzymatic approach has the advantage of obtaining a complex structure polymer which is difficult to be performed in a satisfying way by traditional polymerization methods.

Acknowledgements: We thank Dr. Rosa Erra-Balsells and Dr. Hiroshi Nonami, Ehime University for realization of UV-MALDI-TOF-MS and CONICET for partial financial support.

\section{References and Notes}

1. Kaplan, D.L.; Dordick, J.; Gross, R.A.; Swift, G. in Enzymes in polymer synthesis; Gross, R.A., Kaplan, D.L., Swift, G., Eds.; ACS: Washington, 1996, Chapter 1.

2. Dordick, J.S. TIBTECH 1992, 19, 287.

3. Chaudhary, A.K.; Beckman, E.J.; Russell, A.J. in Enzymes in polymer synthesis, Gross, R.A., Kaplan, D.L., Swift, G., Eds.; ACS: Washington, 1996, Chapter 2. 